\title{
EFECTOS DE LA INTENSIDAD DE LUZ, METODOS DE CONSERVACIÓN Y TIEMPO DE ALMACENAMIENTO EN LA GERMINACIÓN DE Cortón lechleri Muell ARG.
}

\author{
Américo * \\ Oscar Gill Villacorta**
}

\section{RESUMEN}

En Pucallpa-Perú, se realizó un experimento para determinar la germinación de semillas de Croton lechleri en condiciones variadas de intensidad de luz (100, 60 y 30\%), del método de conservación (temperatura ambiente y refrigerado) y del tiempo de almacenamiento $(0,30,60,90,120$ y 150 días $)$, en condiciones de vivero.

Se encontró que 30 y $60 \%$ de luz tienen el mismo efecto en la germinación ( $35 \%$ y $37 \%$ respectivamente), y fueron superiores a los obtenidos con $100 \%$ de luz (23\%). En el método de conservación, la refrigerada fue mejor $(47 \%)$ que la conservación a temperatura ambiente (16\%). La intensidad de luz y el tiempo de almacenamiento tuvieron efecto en la germinación, debido a que las semillas son menos sensibles a la luz a mayor tiempo de almacenamiento. El método de conservación en función del tiempo de almacenamiento expresó que las semillas mantuvieron su viabilidad hasta 150 días bajo refrigeración, pero cuando se almacenó a temperatura ambiente se redujo a 90 días. Es conveniente almacigar las semillas inmediatamente después de haber sido cosechadas o almacenarlas en refrigerador para obtener una germinación aceptable.

\section{ABSTRACT}

In Pucallpa, Perú an experiment was performed in order to determine the gerrnination of Croton lechleri seeds in varied condition of brightness of light (100,60 and 30\%), the method of conservation (normal and refrigerated temperature) and storage time $(0,30,60,90,120$ and 150 days), in greenhouse condition.

It was found that 30 and $60 \%$ of light have the same effect on the germination (35\% and $37 \%$ respectively), and were higher than those obtained with $100 \%$ of 
light $(23 \%)$. In the method of conservation the refrigerated form was better than that of normal temperature $(16 \%)$. The brightness of light and the time of storage had their effect on the germination due to the fact that seeds are less sensitive to light ifkept in storage for a longer time. The method of conservation related to the time of storage expressed that the seeds kept their viability up to 150 days in refrigeration. When $\sim$ a they were stored at normal temperature it was reduced to 90 days. To get an acceptable gerrnination, it is convenient either to keep the seeds inmediately after having been harvested or refrigerate them.

\section{INTRODUCCION}

La gran variedad florística de los bosques amazónicos está seriamente amenazada debido a la depredación a que son sometidas muchas especies de importancia económica; éstas son aprovechadas de manera que no se asegura su sostenibilidad, como la sangre de grado (Croton Lechleri M. Arg.), por ejemplo, que ocupa un sitial preferencial en la lista de las especies forestales.

La sangre de grado es una de las especies cuyas propiedades medicinales son ampliamente conocidas, sin embargo hay un gran desconocimiento para un desarrollo artificial exitoso, lo cual dificulta la producción continua de material reproductivo.

Con base a estas consideraciones, se realizó el estudio titulado "Efecto de la intensidad de luz, método de conservación y tiempo de almacenamiento en la germinación de Croton lechleni M. Arg.", cuyo principal objetivo es el de determinar el efecto de la intensidad de luz, dos métodos de conservación y diferentes períodos de almacenamiento en la germinación de semillas de sangre de grado.

\section{REVISION DE LITERATURA}

\section{A. Generalidades sobre la sangre de grado}

\section{Aspectos generales}

Sangre de grado es el nombre comercial del látex de algunas especies del género Croton, nombre debido a la similitud con el color y la viscosidad de la sangre. Es astringente, produciendo una sensación de sequedad con olor característico a madera (Morales, 1978). 
Etimológicamente el género Croton proviene del griego Kroton, que significa garrapata de perro, haciéndose alusión en Europa a la forma de las semillas (Soukup, 1968).

El uso de sangre de grado es muy difundido en la Amazonía Peruana como planta medicinal cicatrizante de heridas y úlceras, y en el mundo por poseer propiedades curativas para afecciones pulmonares de niños prematuros, existiendo una gran demanda tanto para el mercado nacional como para el extranjero (Phillips, 1991).

\section{Hábitat y distribución}

La sangre de grado es una especie propia de bosques intervenidos, habita también en las riberas de los ríos con suelos ricos en nutrientes, sujetas a inundaciones temporales y tiene una amplia distribución en todos los bosques tropicales (Lao, 1984).

En el Perú se encuentra distribuida en toda la Amazonía, existiendo cuatro especies de sangre de grado identificadas: Croton draconoides, C. erytrochilus, C. Palanostigma y C. lechleri; todas tienen características dendrológicas similares y sus resinas se emplean con propiedades medicinales iguales (Rutter, 1990).

Croton draconoides y Croton lechleri son especies similares, con la diferencia que el primero es originario de Brasil y el segundo del Perú, existiendo otra especie similar que habita en Venezuela, que es el Croton draco (Macbride, 1951 y Encamación, 1983).

\section{B. Dendrología de la especie}

\section{Taxonomía}

Según constancia emitida por el Instituto de Investigaciones de la Amazonia Peruana, la muestra botánica identificada corresponde a la Familia Euphorbiaceae, género Croton y especie Croton lechleri Muell Arg. 


\section{Descripción dendrológica}

Según Macbride (1951) y Lao (1984), Croton lechleri es un árbol monoico de 8 a 20 $\mathrm{m}$ de alto; fuste de 15 a $30 \mathrm{~cm}$ de diámetro; de látex rojizo al cortar; el tipo de raíz es pivotante y ramificado; la forma de la copa es amplia globosa; la corteza externa tiene un color grisáceo blanquecino y la interna un color rosado crema de sabor astringente, textura coriácea fibrosa; las hojas son alternas a veces opuestas o verticiliadas con dos glándulas en la base, 12 a $40 \mathrm{~cm}$ de longitud, 5 a $14 \mathrm{~cm}$ de ancho, tiene una inflorescencia terminal en racimos (espigas), alcanza hasta $30 \mathrm{~cm}$ de longitud, las flores son monoicas de color blanco amarillento, olor astringente; el fruto es una cápsula con tres monocarpos bivalvos elásticamente dehiscente con un promedio de $4,5 \mathrm{~mm}$ de longitud por $3 \mathrm{~mm}$ de ancho; las semillas son pequeñas de color gris oscuro, parecido al dorso de la garrapata, con carúncula y endospermo oleaginoso.

\section{Efecto de la luz en la germinación de las semillas}

Investigaciones sobre el efecto de la luz en la germinación indican que en muchas especies forestales la germinación de sus semillas como el desarrollo inicial, son beneficiados por la exposición a la luz. Esto se debe a que la luz ejerce un efecto estimulante en la germinación de algunas especies, siendo importantes tanto la calidad como la cantidad de luz (Fundeagro, 1991).

Unas pocas especies de plantas tienen semillas que no pueden germinar si no son expuestas a la luz; se incluye en este grupo algunas especies del género Ficus. Otras expuestas a la luz germinan mejor que mantenidas a completa oscuridad; por el contrario, la germinación de algunas especies parece retardarse o inhibirse en presencia de luz (Meyer, 1970).

\section{Almacenamiento}

Hartman y Klester (1962) manifiestan que las condiciones de almacenamiento que mantienen la viabilidad de las semillas son aquellas que hacen más lentas la respiración y otros procesos metabólicos sin dañar el embrión, contando entre los más importantes un contenido de humedad reducido de las semillas, temperatura baja y modificación de la atmósfera de almacenamiento.

Cicafor (1982) menciona que es mejor almacenar las semillas a temperaturas frías, porque reducen la respiración, los demás procesos metabólicos y daños por insectos y hongos, pero cuando no se dispone de un medio refrigerado, hay que guardar las semillas en un lugar seco 
bien ventilado, a temperatura ambiente, fuera del alcance de los roedores e insectos, preferentemente aislado del exterior.

La conservación de las semillas forestales es posible con el conocimiento de las condiciones en que deben almacenarse, no existiendo hasta el momento ningún trabajo de investigación que reporte sobre sangre de grado. Es así que para semillas de azúcar huayo (Hymenea oblongifolia), copaiba (Copaifera officinalis) y estoraque (Myroxilon balsamurn) se determinó que deben almacenarse en un ambiente refrigerado a $5^{\circ} \mathrm{C}$, ishpingo (Amburana cearensis) a una temperatura de $15^{\circ} \mathrm{C}$, y para semillas de las especies como aguano masha (Huberodendron swietenoides), caoba (Swietenia macrophylla), cedro (Cedrela odorata), huimba (Ceiba samauma), pashaco quillosisa (Schizolobium sp) y bolaina blanca (Guazuma crinita) es mejor un almacenamiento a temperatura ambiente, necesitando esta última de dos meses de almacenamiento para alcanzar su mayor capacidad germinativa (Colán, 1992).

\section{MATERIALES Y METODOS}

\section{A. Descripción del lugar de estudio}

\section{Ubicación}

El experimento se realizó en la Estación Experimental del Centro Regional de Investigación (CRI) de Ucayali, del Instituto de Investigaciones de la Amazonía Peruana, localizado en el km 12,4 de la carretera Federico Basadre, Pucallpa, Perú.

\section{Ecología y clima}

Según la clasificación de Holdrige, la zona corresponde a la formación ecológicá de Bosque Húmedo Tropical y Bosque muy Húmedo Premontano Tropical. La temperatura media anual es de $25,42^{\circ} \mathrm{C}$, junto con una precipitación promedio anual de $1773,44 \mathrm{~mm}$ y una humedad relativa de $84,47 \%$ (Baldoceda, 1993).

\section{B. Manejo del experimento}

El proyecto consistió en evaluar el porcentaje de germinación de las semillas de sangre de grado, con diferentes intensidades de luz (100, 60 y 30\%), dos métodos de conservación (medio ambiente y refrigerado) y a diferentes períodos de almacenamiento, hasta 150 días con intervalos de 30 días, respectivamente. 


\section{Diseño experimental y factores estudiados}

Se utilizó un diseño experimental de bloques completos al azar con un arreglo factorial de $3 \times 2 \times 6$, distribuidos en parcelas divididas; los tratamientos se dividieron tres veces (Vásquez, 1990).

\section{Características del experimento}

\section{a. Tamaño y características de la parcela}

La parcela tuvo una dimensión de 0,25 $\mathrm{m}$ de ancho por 0,25 $\mathrm{m}$ de largo, construida con tablas de bolaina de $10 \mathrm{~cm}$ de ancho, en las cuales se sembraron 100 semillas de sangre de grado, a un distanciamiento de $2 \mathrm{~cm}$; la separación entre parcelas fue de $5 \mathrm{~cm}$.

\section{b. Tamaño y características del bloque}

La dimensión del bloque fue de 4,50 $\mathrm{m}$ de largo por 0,50 $\mathrm{m}$ de ancho, conteniendo 36 parcelas con una separación de $2 \mathrm{~m}$ entre ellas y un área experimental de 24,75 $\mathrm{m}^{2}$, sembrándose 10800 semillas en las 108 unidades experimentales.

\section{Cuadro 1. Factores estudiados y sus correspondientes niveles.}

\begin{tabular}{|c|c|c|}
\hline Factores & Niveles & Símbolo \\
\hline A. Intensidad & $\begin{array}{l}\text { - } 100 \% \text { de luz } \\
\text { - } 60 \% \text { de luz } \\
\text { - } 30 \% \text { de luz }\end{array}$ & $\begin{array}{l}\mathrm{A}_{1} \\
\mathrm{~A}_{2} \\
\mathrm{~A}_{3}\end{array}$ \\
\hline B. Método de coservación & $\begin{array}{l}\text { - Medio ambiente } \\
\text { - Refrigerado }\end{array}$ & $\begin{array}{l}\mathrm{B}_{1} \\
\mathrm{~B}_{2}\end{array}$ \\
\hline 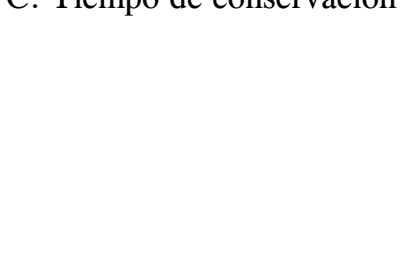 & $\begin{array}{l}\text { - } \text { Cero días } \\
\text { - } 30 \text { días } \\
\text { - } 60 \text { días } \\
\text { - } 90 \text { días } \\
\text { - } 120 \text { días } \\
\text { - } 150 \text { días }\end{array}$ & $\begin{array}{l}\mathrm{C}_{1} \\
\mathrm{C}_{2} \\
\mathrm{C}_{3} \\
\mathrm{C}_{4} \\
\mathrm{C}_{5} \\
\mathrm{C}_{6}\end{array}$ \\
\hline
\end{tabular}




\section{Procedimientos}

\section{Recolección de muestras botánicas}

Las muestras; constituidas por hojas, flores y frutos, fueron recolectadas de 15 árboles ubicados en el km 8 de la carretera Marginal von Humboldt . Sungaroyacu.

\section{Recolección de frutos y obtención de semillas}

Los frutos se colectaron utilizando el método de cosecha de árboles en pie, debido a las características dehiscentes del fruto.

\section{Almacenamiento}

Antes deponer las semillas puras en almacenamiento, primero se acondicionaron envolviéndolas con papel secante en paquetes de 100 semillas y puestas en una bolsa plástica para facilitar su manipuleo, luego fueron introducidas en un envase de vidrio herméticamente cerrado para evitar los cambios de humedad de la semilla al contacto con la humedad relativa del aire (Patiño et al, 1983).

\section{a. Refrigerado}

Las semillas preparadas fueron depositadas en un refrigerador cuya temperatura varió entre $6^{\circ} \mathrm{C}$ a más-menos $4^{\circ} \mathrm{C}$, dependiendo del clima, registrándose la temperatura mínima a las 6 horas y la máxima a las 14 horas con $2^{\circ} \mathrm{C}$ y $10^{\circ} \mathrm{C}$, respectivamente.

\section{b. Temperatura ambiente}

Como en el caso anterior, las semillas fueron puestas en el almacén a temperatura ambiente.

\section{Preparación de las camas de almácigo}

Se hicieron tres camas de $1 \mathrm{~m}$ por 4,5 $\mathrm{m}$, a una altura de $0,20 \mathrm{~m}$ del suelo, en cada cama se puso tres cajas de madera, especialmente preparadas para este experimento, con una dimensión de $0,50 \mathrm{~m} \times 1,50 \mathrm{~m}$ cada una. Cada caja tenía doce compartimientos de $0,25 \mathrm{~m} \times$ $0,25 \mathrm{~m}$. 


\section{Preparación del sustrato y llenado de bolsas}

Para la preparación del sustrato se utilizó tierra negra, arena lavada de río y humus de lombriz, en una proporción de 2:1:1.

\section{Construcción del tinglado}

El tinglado se construyó usando bastidores de madera de $5 \mathrm{~cm} \times 5 \mathrm{~cm}$, con una altura de 1 metro del suelo, en los que se clavó tiras de madera a una distancia de 1,5 ni.

Al primer nivel de luz (100\%) no se le colocó tinglado, en los dos siguientes niveles (60 y $30 \%$ ) se cubrió con mallas negras de plástico graduado, de acuerdo con la intensidad deseada marcada por el fotómetro.

\section{Siembra}

Una vez llenadas las cajas de almácigo con el sustrato, se procedió a efectuar la siembra previo riego de las camas. La operación de la siembra se efectuó presionando las semillas, de tal manera que éstas quedaron enterradas a ras del suelo. Se espolvoreó sobre ellas con el sustrato para evitar la dispersión por efecto del riego; el distanciamiento entre semillas fue de $2 \mathrm{~cm}$. La operación de siembra se efectuó cada 30 días de acuerdo con el diseño, iniciando la misma el 12 de octubre de 1993. Antes de efectuar la siembra se desinfectó las camas. El experimento tuvo una duración de 150 días en la fase de campo.

\section{Evaluación de las variables estudiadas}

\section{Porcentaje de germinación}

El control del porcentaje de germinación de las semillas se realizó diariamente en formatos especiales, desde el inicio de la germinación hasta el momento que dejó de germinar por tres días consecutivos.

El porcentaje de germinación se calculó mediante la siguiente fórmula:

$$
\% \text { de germinación }=\frac{\mathrm{N}^{\mathrm{o}} \text { de semillas }}{\mathrm{N}^{\mathrm{o}} \text { semillas sembradas }} \times 100
$$




\section{E. Análisis estadístico}

Los datos se analizaron bajo un diseño de Bloques Completamente al Azar con arreglo en parcelas divididas de $3 \times 2 \times 6$, las mismas que se muestran en el Cuadro 2. Para comparar la diferencia entre promedios se utilizó la prueba de Tuckey $(\mathrm{P} \leq 0.05)$.

Cuadro2. Análisis de variación para la germinación de semillas de sangre de grado.

\begin{tabular}{lcc}
\hline Fuentes de variación & G.L \\
\hline Fuentes de variación & $(\mathrm{r}-1)$ & 2 \\
Bloques & $(\mathrm{a}-1)$ & 2 \\
A (Intensidad de luz) & $(\mathrm{a}-1)(\mathrm{r}-1)$ & 4 \\
Error (a) & $(\mathrm{b}-1)$ & 1 \\
B (Método de conservación) & $(\mathrm{a}-1)(\mathrm{b}-1)$ & 2 \\
A x B & $\mathrm{a}(\mathrm{r}-1)(\mathrm{b}-1)$ & 6 \\
Error (b) & $(\mathrm{c}-1)$ & 5 \\
C (Tiempo de almacenam.) & $(\mathrm{a}-1)(\mathrm{c}-1)$ & 10 \\
A x C & $(\mathrm{b}-1)(\mathrm{c}-1)$ & 5 \\
B X B x C & $(\mathrm{a}-1)(\mathrm{b}-1)(\mathrm{c}-1)$ & 10 \\
Error (c ) & $\mathrm{ab}(\mathrm{r}-1)(\mathrm{c}-1)$ & 60
\end{tabular}

\section{RESULTADOS Y DISCUSION}

A. Efecto de los factores estudiados en la germinación de semillas de sangre de grado

Según los resultados obtenidos se observa que la mayor germinación se presentó con intensidades de luz de 30 y $60 \%$, en semillas conservadas refrigeradas durante 150 días, mientras que con semillas conservadas al 
ambiente sólo hubo germinación hasta los 60 días, observándose que con este método el porcentaje de germinación desciende desde los 30 días (Cuadro 3).

Cuadro 3. Germinación de Croton lechleri por efecto de la intensidad de luz, método de conservación y tiempo de almacenamiento. Pucallpa, Perú,1994.

\begin{tabular}{|c|c|c|c|c|c|c|}
\hline \multicolumn{2}{|c|}{ Intensidad de Luz } & \multicolumn{5}{|c|}{ Tiempo de almacenamiento (días) } \\
\hline$(\%)$ & 0 & 30 & 60 & 90 & 120 & 150 \\
\hline \multicolumn{7}{|c|}{$\begin{array}{c}\text { Conservación en medio ambiente } \\
\text { Germinación }(\%)\end{array}$} \\
\hline 100 & 28 & 15 & 14 & 0 & 0 & 0 \\
\hline 60 & 69 & 26 & 23 & 0 & 0 & 0 \\
\hline 30 & 61 & 28 & 32 & 0 & 0 & 0 \\
\hline \multicolumn{7}{|c|}{$\begin{array}{c}\text { Conservación refrigerada } \\
\text { Germinación }(\%)\end{array}$} \\
\hline 100 & 26 & 21 & 35 & 50 & 36 & 59 \\
\hline 60 & 56 & 41 & 52 & 50 & 62 & 60 \\
\hline 30 & 63 & 41 & 60 & 46 & 35 & 53 \\
\hline
\end{tabular}

De acuerdo con los resultados del análisis de varianza, la germinación de las semillas de Croton lechleri mostraron diferencias altamente significativas por efectos de la intensidad de luz, métodos de conservación y tiempo de almacenamiento. La interacción intensidad de luz por método de almacenamiento ( $\mathrm{A} \times \mathrm{B}$ ) y método de conservación por tiempo de almacenamiento (B x C) tuvieron diferencias altamente significativas (Cuadro 4).

\section{Efecto de la intensidad de luz}

Según los resultados obtenidos, el mayor porcentaje de germinación se obtuvo cuando las semillas se almacigaron con intensidades de luz de 30 y $60 \%$. 
Cuadro 4. $\quad$ ANVA del número de semillas de Croton lechleri germinadas por efecto de la densidad de luz, método de conservación y tiempo de almacenamiento. Pucallpa, Perú, 1994

\begin{tabular}{|c|c|c|c|}
\hline Fuentes de variación & G.L & C.M & Fc. \\
\hline Repeticiones & 2 & 83,67 & $2,47 \mathrm{NS}$ \\
\hline Intensidad de luz (A) & 2 & 1830,71 & $54,05 * *$ \\
\hline Error (a) & 4 & 33,87 & \\
\hline Método conservación (B) & 1 & 25269,48 & $610,67 * *$ \\
\hline$A \times B$ & 2 & 76,71 & $1,69 \mathrm{NS}$ \\
\hline Error (b) & 6 & 41,38 & \\
\hline Tiempo almac. (C) & 5 & 1933,57 & $26,97 * *$ \\
\hline $\mathrm{C} \times \mathrm{A}$ & 10 & 456,65 & $6,98 * *$ \\
\hline $\mathrm{C} \times \mathrm{B}$ & 5 & 2567,93 & $35,99 * *$ \\
\hline \multirow[t]{2}{*}{ Error $(\mathrm{C})$} & 10 & 90,31 & $1,26 \mathrm{NS}$ \\
\hline & 60 & 71,70 & \\
\hline
\end{tabular}

Total 107 578,74

** $=$ Altamente significativo

$\mathrm{CVa}=18,38$

$\mathrm{CVb}=20,27$

Los promedios de germinación de los tratamientos con una intensidad de luz de 30 y $\mathbf{6 0} \%$ no presentan diferencias significativas pero son superiores al obtenido con intensidad del 100\% de luz (Cuadro 5), debido a que las semillas frescas son más sensibles a intensidades altas de luz, las mismas que producen temperaturas altas que pueden dañar al embrión o se vuelven latentes, denominado por Hartman y Kester (1962) como daño del calor. 
Cuadro 5. Prueba de Tuckey para la intensidad de luz, Pucallpa, Perú 1994.

\begin{tabular}{ccc}
\hline \% de luz & \% de germinación & Tuckey 0,05 \\
\hline 100 & 23,56 & $\mathrm{~b}$ \\
60 & 36,61 & $\mathrm{a}$ \\
30 & 35,06 & $\mathrm{a}$
\end{tabular}

$\mathrm{ALS}=\quad 9,13$

\section{Efecto del método de conservación}

El método de conservación tuvo un efecto altamente significativo en la germinación de las semillas de sangre de grado, demostrando que el mejor método para la conservación de las semillas de esta especie es el método refrigerado (Cuadro 4).

La prueba de Tuckey para el método de conservación muestra que las semillas de sangre de grado conservadas en refrigeradora son superiores a las conservadas al ambiente (Cuadro 6), debido fundamentalmente a que la respiración y Otros procesos metabólicos en la conservación refrigerada son más lentos, al estar sometidos a temperaturas bajas, manteniendo a la semilla en estado latente (Hartman y Kester, 1962), contrariamente a las conservadas al ambiente, donde los cambios de temperatura pueden haber acelerado el oxidamiento de la estructura oleaginosa de estas semillas (Vidal, 1984).

Cuadro 6. Prueba de Tuckey para el método de conservación en, Pucallpa, Perú, 1994.

\begin{tabular}{ccc}
\hline Conservación & \% de germinación & Tuckey $\mathbf{0 , 0 5}$ \\
\hline Refrigerado & 47,04 & $\mathrm{~b}$ \\
Medio ambiental & 16,44 & $\mathrm{a}$ \\
& & \\
\hline
\end{tabular}

$\operatorname{ALS}=4,70$ 


\section{Efecto del tiempo de almacenamiento}

En el Cuadro 7 se observa la prueba de Tuckey ( $\mathrm{p} \leq$ OO.O5) para el tiempo de almacenamiento. Al inicio del experimento se obtuvo un mayor porcentaje de germinación y superior a los demás tiempos de almacenamiento. A los $30,60 \mathrm{y}$ 150 días no hubo diferencias en los promedios de los porcentajes de germinación, obteniéndose los menores resultados de germinación a los 90 y 120 días de almacenamiento, lo cual está relacionado con la nula germinación encontrada con semillas almacenadas a temperatura ambiente en los mismos períodos de evaluación.

\begin{tabular}{ccc}
\hline $\begin{array}{c}\text { Tiempo de almac. } \\
\text { (días) }\end{array}$ & \% de germinación & $\begin{array}{c}\text { Prueba Tuckey } \\
\mathbf{p} \leq \mathbf{0 , 0 5}\end{array}$ \\
\hline 0 & 50,50 & $\mathrm{a}$ \\
30 & 28,67 & $\mathrm{~b}$ \\
60 & 36,06 & $\mathrm{~b}$ \\
90 & 24,33 & $\mathrm{c}$ \\
120 & 22,06 & $\mathrm{c}$ \\
150 & 28,83 & $\mathrm{~b}$ \\
& & \\
\hline
\end{tabular}

$\mathrm{ALS}=8,30$

4. Interacción intensidad de luz por tiempo de almacenamiento (A x C).

De acuerdo con el ANVA (Cuadro 4), la interacción intensidad de luz por tiempo de almacenamiento (A x C) resultó ser altamente significativa, lo cual indica que la intensidad de luz y el tiempo de almacenamiento actúan simultáneamente en la germinación de las semillas de sangre de grado

En la figura 1, se observa que a partir de los 60 días de almacenamiento el porcentaje de germinación de los niveles del 30 y $60 \%$ disminuye hasta igualarse con el nivel del $\mathbf{1 0 0 \%}$ de intensidad de luz a los 90 días, motivado por la pérdida de la viabilidad de las semillas conservadas al ambiente. 
Figura 1. Germinación de Croton lechleri por efecto de la intensidad de luz y tiempo de almacenamiento.

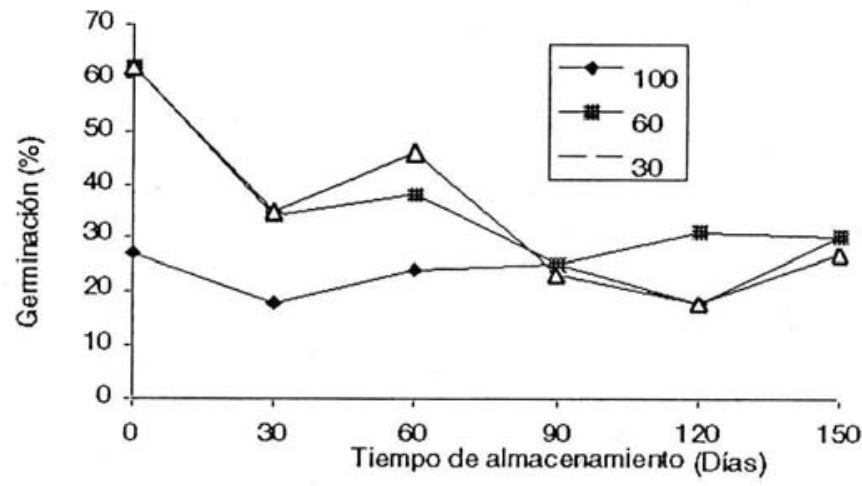

Según el ANVA de los efectos simples de la interacción intensidad de luz por tiempo de almacenamiento $(\mathrm{A} \times \mathrm{C}$ ), se encontró diferencias altamente significativas entre el tiempo de almacenamiento (C) y todos los niveles de intensidad de luz. Para la intensidad de luz se encontró alta significación estadística sólo en los niveles $\mathrm{C}_{1}$ (cero días), $\mathrm{C}_{2}$ (30 días), $\mathrm{C} 3$ (60 días) y $\mathrm{C}_{5}$ (120 días), no así para los niveles $\mathrm{C}_{4}$ (90 días) y $\mathrm{C}_{6}$ (150 días); esto indica que en estos dos últimos niveles el factor intensidad de luz no ha afectado el porcentaje de germinación, por lo cual se observa una tendencia a igualarse.

\section{Interacción método de conservación por tiempo de almacenamiento (B x C).}

La interacción método de conservación por tiempo de almacenamiento $(\mathrm{B} \times \mathrm{C})$ resultó ser altamente significativa (Cuadro 4), lo cual implica que ambos factores actúan conjuntamente en la germinación.

A medida que aumenta el tiempo de almacenamiento, mayor es la diferencia por influencia del método de conservación. Las semillas conservadas al ambiente descienden hasta llegar a cero $(0 \%$ de germinación a los 90 días, mientras que las semillas conservadas refrigeradas mantienen el poder germinativo hasta los 150 días (Figura 2). 
Figura 2. Germinación de Croton lechleri por efecto del tiempo de almacenamiento el método de conservación.

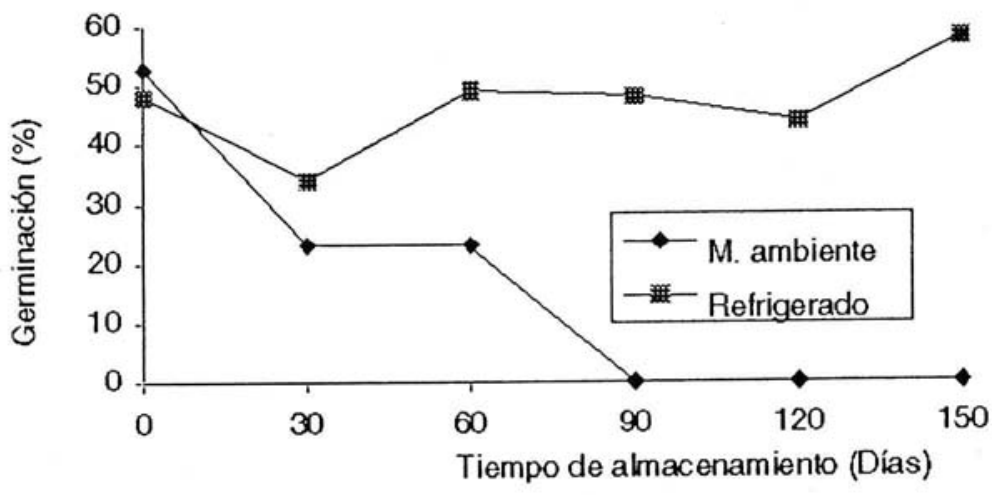

Tiempo de almacenamiento (días)

\section{CONCLUSIONES}

Del análisis de los resultados se concluye que:

1. El efecto de la intensidad de luz en la germinación de las semillas de sangre de grado (Croton lechleri), fue estimular el proceso de germinación, alcanzando los mejores niveles de germinación con intensidades de luz de 30 y $60 \%(35,05$ y $36,61 \%$ de germinación, respectivamente), contra la intensidad del $100 \%$ de luz, que fue el más bajo con una germinación de $23,56 \%$.

2. El efecto del método de conservación radica en mantener viables las semillas por un período mayor, siendo el mejor método para conservar las semillas de sangre de grado el refrigerado, con $47 \%$ de germinación contra $16,44 \%$ con el método de conservación al ambiente.

3. Las semillas de sangre de grado conservadas al ambiente perdieron su viabilidad a los 60 días, mientras que conservadas en refrigeración mantuvieron su viabilidad en más del $\mathbf{5 0} \%$ en un período de 150 días. La temperatura en este método, entre 2 y $10^{\circ} \mathrm{C}$, es la que puede mantener viables las semillas por un período mayor. 


\section{RECOMENDACIONES}

Se recomienda lo siguiente:

1. Almacigar las semillas de Croton lechleri con intensidades de luz de 30 y $60 \%$.

2. Conservar semillas de sangre de grado en un ambiente refrigerado.

3. Realizar un estudio sobre almacenamiento de semillas de sangre de grado por períodos mayores de 150 días en medio refrigerado.

\section{BIBLIOGRAFIA}

BALDOCEDA, R. 1993. Diagramas bioclimáticos de /a zona de Pucallpa y Atalaya. Pucallpa: Facultad de Ciencias Forestales. Universidad Nacional de Ucayali. Perú. 21 pp.

CENTRO DE INVESTJGACION Y CAPACJTACION FORESTAL. 1982. La investigación realizada en el Cicafor, Cajamarca 1976-1980. Cajamarca: Proyecto Específico Forestal No 01. Perú. 102 pp.

COLAN, V. 1992. Manejo de semillas de diez especies forestales en el Bosque Nacional Alexander von Humboldt. Lima: Proyecto Suelos Tropicales. IINIA. Perú. 33 pp.

ENCARNACJON, E. 1983. Nomenclatura de las especies forestales en el Perú. Documento de trabajo $N^{\circ}$ 7. Lima (Perú): Proyecto PNUD/FAO/PER]8 1/002. Perú. 80 pp.

FUNDACION PAPA EL DESARROLLO DEL AGRO. 1991. Manual de control de calidad de semillas. Lima (Perú): Impreso en Elite Gráfica 5. A. 238 pp.

HARTMAN, T. y KESTER, E. 1962. Propagación de plantas. México. 693 pp.

LAO, R. 1984. Descripción dendrológica de especies forestales en el Asentamiento Rural Forestal Alexander von Llumholdt. Huánuco (Perú): Proyecto Especial Pichis Palcazu. $71 \mathrm{pp}$.

MACBRIDE, F. 1951. Flora of Perú. En: Field Museum of Natural History. Vol. XIII Part IIIA. October 117. United States 55O $p$. 
MEYER, B. 1970. Introducción a la Fisiología Vegetal. Buenos Aires (Argentina): Editorial Universitaria de Buenos Aires. 579 pp.

MORALES, G. 1978. Sangre de grado, maravilloso cicatrizante. En: El Comercio. Lima.

PATIÑO, V; VILLAGOMEZ, Y; DE LA GARZA, P; TALAVERA, 1; y CAMACHO, F.1983. Guía para la recolección y manejo de semillas de especies forestales. México: Instituto Nacional de Investigaciones Forestales. $180 \mathrm{pp}$.

PHILLIPS, 0. 1991. La sangre de grado en el Departamento de Madre de Dios. Informe entregado a la Compañía Farmacéutica Shaman Inc. 8 pp.

RUTTER, R. 1990. Catálogo de plantas útiles de la Amazonía Peruana. Pucallpa (Perú): Instituto Lingüístico de Verano. 568 pp.

SOUKUP, J. 1968. Vocabulario de los nombres vulgares de la flora peruana y catálogo de los géneros. Lima (Perú): Editorial Salesiano. 332 pp.

VASQUEZ, V. 1990. Experimentación agrícola. Primera edición. Lima (Perú): Instituto Nacional de Desarrollo. 246 pp.

VIDAL, J. 1984. Curso de botánica. Buenos Aires (Argentina): Editorial Stella Viamonte. 548 pp. 